\title{
Balanço entre ácidos graxos ômega-3 e 6 na resposta inflamatória em pacientes com câncer e caquexia
}

\section{Omega-3 and 6 fatty acids balance in inflammatory response in patients with cancer and cachexia}

Adriana GARÓFOLO ${ }^{1,2}$

Antônio Sérgio PETRILLI ${ }^{3}$

\section{RE S U M O}

O emagrecimento, associado à perda de massa magra, é um fenômeno observado com freqüência em pacientes com câncer. Tal condição predispõe o paciente ao maior risco de infecções, pior resposta aos tratamentos implantados e, como conseqüência, desfavorece o prognóstico de cura. Além disso, a desnutrição também está associada à pior qualidade de vida. Dessa forma, algumas terapias têm sido propostas na tentativa de reverter o catabolismo, por meio da atenuação da resposta inflamatória, observado em grande porcentagem de pacientes com câncer e caquexia. Entre elas, a suplementação com ácidos graxos da família ômega-3 pode representar uma estratégia na redução da formação de citocinas pró-inflamatórias, favorecendo a tolerância metabólica dos substratos energéticos e atenuando o catabolismo protéico, com o intuito de melhorar o prognóstico de cura de pacientes com câncer. Entretanto, os estudos mostram alguns resultados conflitantes da suplementação com ômega-3 na resposta imunológica. Por outro lado, em pacientes com câncer, os ensaios clínicos mostraram atenuar a resposta inflamatória e melhorar o estado nutricional. O objetivo deste artigo é realizar uma revisão criteriosa do assunto.

Termos de indexação: ácidos graxos; caquexia; desnutrição; inflamação; neoplasias.

A B S T R A C T

Emaciation and loss of lean body mass is a frequent phenomenon observed in cancer patients. This condition leads to infection risk and a poor response to treatment, thus reducing the chances of cure. Furthermore,

\footnotetext{
1 Pós-graduanda da Disciplina de Nutrologia e Metabolismo, Departamento de Pediatria, Universidade Federal de São Paulo. São Paulo, SP, Brasil.

2 Setor de Nutrologia e da Equipe Multidisciplinar de Terapia Nutricional, Instituto de Oncologia Pediátrica, Universidade Federal de São Paulo. Rua Botucatu, 743, 04023-062, São Paulo, SP, Brasil. Correspondência para/Correspondence to: A. GARÓFOLO. E-mail: <adrigarofolo@hotmail.com>.

3 Instituto de Oncologia Pediátrica, Departamento de Pediatria, Universidade Federal de São Paulo. São Paulo, SP, Brasil.
} 
malnutrition is also associated with a poor quality of life. Therefore, therapies have been proposed in attempt to revert the catabolism observed in most of these patients by attenuating the inflammatory response. Among them, omega-3 fatty acid supplementation may be a strategy to reduce the production of pro-inflammatory cytokines and improve metabolic substrate tolerance, decreasing protein catabolism in order to ameliorate the prognosis of cure in cancer patients. However, studies demonstrate some conflicting results of ômega-3 supplementation on immune response. On the other hand, clinical trials in cancer patients demonstrate that the inflammatory response decreases and the nutritional status improves. The aim of this paper is to elaborate a strict review of the subject.

Indexing terms: fatty acids; cachexia; malnutrition; inflammation; neoplasms.

\section{N T R O D U ÇÃ O}

A perda de peso e a desnutrição são os distúrbios nutricionais mais freqüentemente observados em pacientes com câncer ( $40 \%$ a $80 \%$ dos casos) ${ }^{1}$, sendo que até $30 \%$ dos pacientes adultos apresentam perda superior a $10 \%$ do peso ${ }^{2}$. Em crianças, a prevalência de desnutrição aproxima-se de $30 \%$ a $35 \%$, sendo mais freqüente entre portadores de tumores sólidos ${ }^{3}$.

Dos fatores associados à desnutrição, destacam-se, principalmente, a ingestão alimentar, que é influenciada pelo tumor e pela presença de várias substâncias tóxicas utilizadas no tratamento oncológico, as alterações no metabolismo energético e dos nutrientes e o aumento nas perdas nutricionais, devido a vômitos, má-absorção, diarréia e falência renal ${ }^{4-8}$.

O déficit do estado nutricional, principalmente quando evidenciado pela perda de massa corporal magra, está associado ao aumento no tempo de hospitalização e na morbimortalidade? ${ }^{9}$. Tal associação decorre do fato de que um aumento percentual na perda de massa magra está diretamente associado ao aumento de fatores de co-morbidade. Entre eles, redução da imunidade, com aumento de infecções, prejuízos nos processos de cicatrização, fraqueza muscular, pneumonia e morte ${ }^{10}$. Alguns estudos demonstraram redução na atividade das células NK (Natural Killer) e na relação Thelper/Tsupressor (CD4/CD8), em pacientes desnutridos com câncer ${ }^{11,12}$. Além disso, Gogos et al. ${ }^{12}$ demonstraram um impacto negativo na sobrevida de pacientes desnutridos com câncer, que foi menor em comparação aos eutróficos.
Nos últimos anos, diferentes abordagens no tratamento nutricional têm sido utilizadas para promover uma alimentação capaz de corrigir os déficits observados em pacientes com câncer: orientação dietética, suplementos orais, nutrição enteral e parenteral e estimulantes do apetite ${ }^{13}$.

Atualmente, a utilização de nutrientes imunomoduladores, associada a nutrientes antioxidantes, com a finalidade de estabilizar o catabolismo e reduzir os danos peroxidativos, tem demonstrado resultados promissores. Com o objetivo de realizar uma revisão sobre o balanço dos ácidos graxos ômega-3 e 6 na resposta inflamatória, em pacientes com câncer e caquexia, propôs-se este estudo. Nos parágrafos seguintes, serão abordados a resposta metabólica e os efeitos da suplementação com ômega-3 em pacientes com câncer. Esta revisão pesquisou ensaios clínicos e estudos de revisão nas bases Medline, considerando os artigos publicados nos últimos 15 anos.

\section{Resposta metabólica do hospedeiro ao tumor maligno}

A presença do tumor maligno, freqüentemente, induz à perda de peso, que está associada ao aumento na síntese de mediadores imunológicos de resposta pró-inflamatória e produção de fatores que induzem à degradação protéica $^{14}$

Entre eles, os mais importantes são o Fator de Necrose Tumoral (TNF- $\alpha$ ), as Interleucinas-1 e 6 (IL1 e IL6) ${ }^{15}$ e o Fator Indutor de Proteólise (PIF) ${ }^{16}$. Além da redução do apetite, o TNF- $\alpha$ e as IL1 e 
IL6 estimulam as respostas metabólicas de fase aguda, com aumento de proteínas positivas, como a proteína-C reativa (PCR), fibrinogênio, $\alpha$-antitripsina, ceruloplasmina, glicoproteína e haptoglobina, e com redução das proteínas negativas, como albumina, pré-albumina e transferrina.

Essa resposta leva a alterações no metabolismo de nutrientes, que se caracterizam pelo aumento dos triglicérides circulantes, apesar da maior utilização de ácidos graxos, intolerância à glicose e catabolismo protéico acentuado ${ }^{4,6}$. Também ocorre aumento na taxa metabólica basal, com conseqüente aumento no gasto energético ${ }^{8,14}$.

Além disso, o PIF estimula a proteólise, levando à perda de massa muscular ${ }^{16}$. Todas essas alterações são responsáveis pelo quadro de emagrecimento e caquexia, presente num elevado percentual de pacientes com câncer ${ }^{17}$.

Achados recentes mostram que a presença de uma resposta inflamatória é capaz de identificar pacientes com doença maligna mais agressiva. Além disso, essa condição inflamatória também pode comprometer a farmacocinética e farmacodinâmica das drogas antineoplásicas. O aumento de alguns mediadores inflamatórios e de proteínas marcadoras da resposta inflamatória demonstrou ser fator prognóstico independente da sobrevida em vários tipos de neoplasias malignas, incluindo melanoma, mieloma, carcinoma renal, câncer de ovário e cânceres do trato gastrintestinal. Acredita-se, portanto, que a resposta inflamatória é um fator potencialmente importante na variabilidade interindividual da quimioterapia quanto à resposta ao câncer e seus efeitos tóxicos ${ }^{18}$.

Dessa forma, estratégias terapêuticas para atenuar a resposta inflamatória aguda exacerbada, sejam elas medicamentosas ou nutricionais, devem ser exploradas na tentativa de melhorar as chances de cura de pacientes com câncer. Entre as estratégias nutricionais atuais mais estudadas está o balanço entre a ingestão de lipídeos da família ômega-3 e ômega-6.

\section{Papel dos lipídeos na regulação da resposta inflamatória}

Atualmente, a adequação do balanço dietético de lipídeos tem motivado inúmeras investigações. Em pacientes com alterações das respostas metabólicas, o equilíbrio entre os lipídeos da dieta tem como propósito controlar a resposta inflamatória exacerbada, por meio da relação entre os tipos de ácidos graxos polinsaturados ingeridos ${ }^{19}$.

Existem três famílias importantes de ácidos graxos comumente consumidos na dieta: $\omega-9, \omega$ 6 e $\omega-3$ (Figura 1), sendo que apenas as duas últimas representam os ácidos graxos essenciais para o organismo. Os lipídeos de 18 átomos de carbonos que pertencem a essas famílias - ácido $\alpha$-linolênico (18:3 $\omega$-3), ácido linoléico (18:2 $\omega-6)$ e ácido oléico (18:1 $\omega$-9) - usam as mesmas enzimas - dessaturases ( $\Delta 6$ e $\Delta 5$ ) e uma elongase - para sintetizar seus derivados com 20 átomos de carbonos: ácido eicosapentaenóico (EPA) (20:5 $\omega$-3), ácido araquidônico (AA) (20:4 $\omega$-6) e ácido eicosatrienóico (ETA) (20:3 $\omega$-9). Em ordem de preferência, os substratos para essas enzimas são: $\omega-3>\omega-6>\omega-9$ (Figura 2). Entretanto, existem duas classes de lipídios essenciais para a síntese dos eicosanóides: $\omega$-3 e $\omega-6$, por meio dos seus derivados ácidos eicosapentaenóico e araquidônico ${ }^{20}$. Assim, devido à importância do balanço entre os ácidos graxos das famílias 3 e 6 na resposta inflamatória, estas serão abordadas na discussão deste texto.

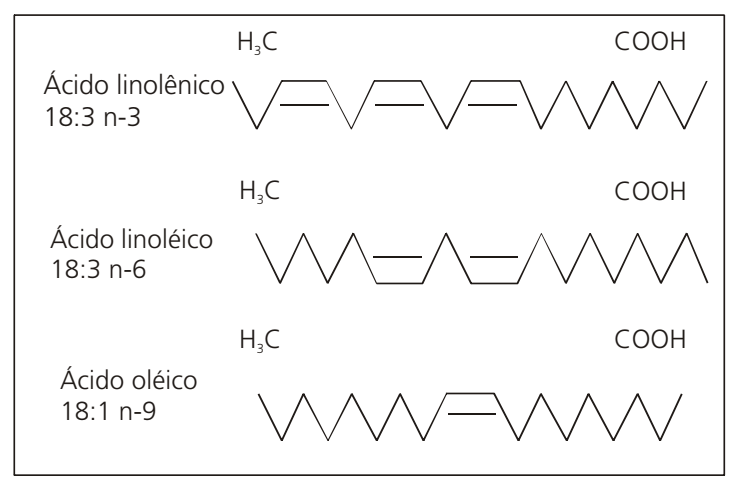

Figura 1. Estrutura dos ácidos graxos das famílias $\omega-3, \omega-6$ e $\omega-9$. 


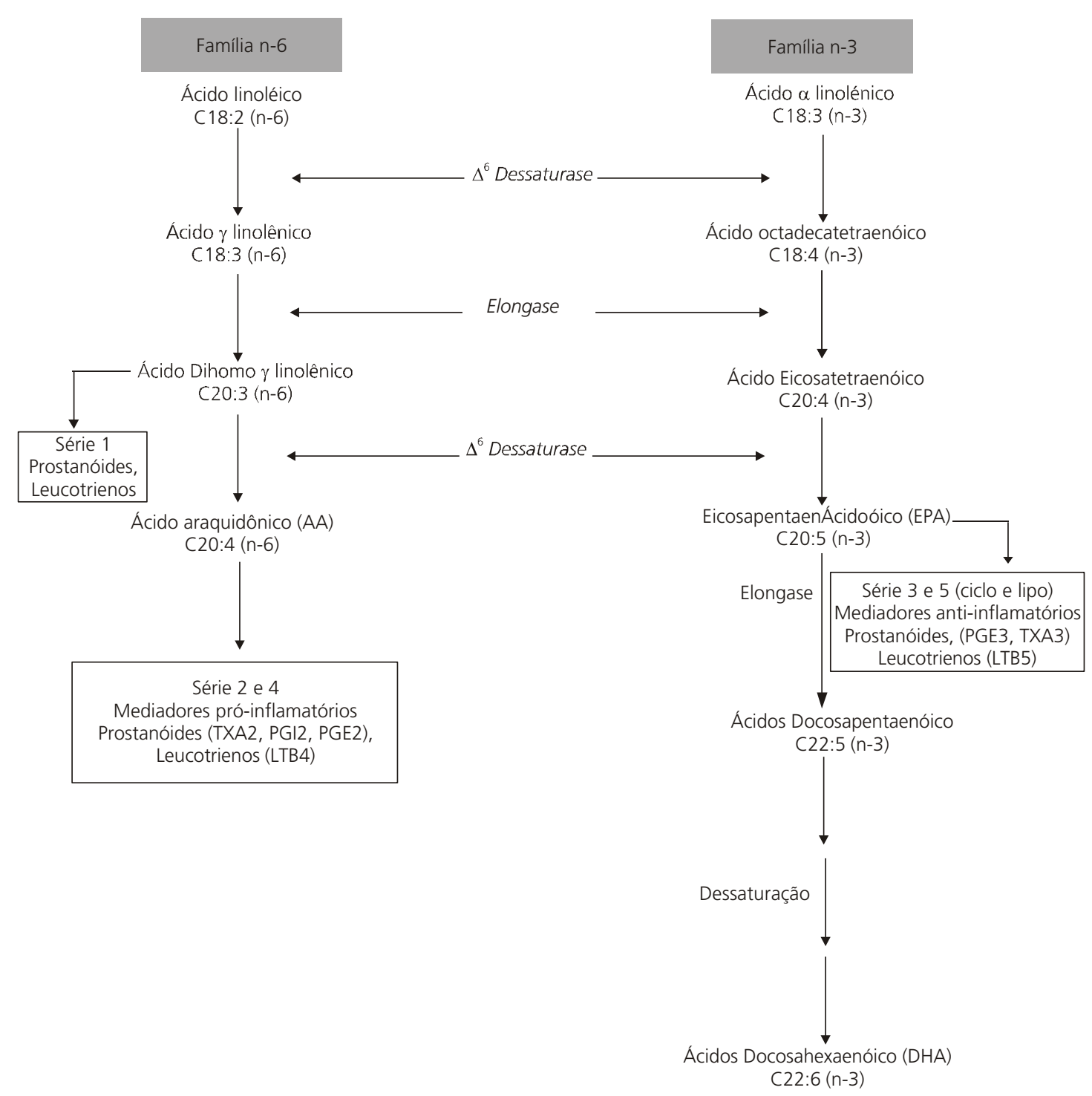

Figura 2. Esquematização da via de biossíntese dos ácidos graxos poliinsaturados. Adaptado de Calder ${ }^{18}$.

A dieta consumida atualmente pela população do ocidente, conhecida como dieta ocidental, é rica em ácido linoléico $(\omega-6)$, presente, entre outros, nos óleos de milho, girassol e soja ${ }^{21}$. Em uma dieta Norte Americana típica, por exemplo, consome-se $89 \%$ do total de ácidos graxos poliinsaturados como ácido linoléico, enquanto $9 \%$ de ácido linolênico ${ }^{22}$. O alto consumo implica no aumento da relação $\omega-6: \omega-3$, principalmente quando a ingestão de peixe ou de óleo de peixe é baixa. Segundo Fürst $^{23}$, entre as civilizações modernas do Ocidente, essas dietas apresentam uma relação $\omega-6: \omega-3$ de 16,7:1. Esse perfil é des- favorável, especialmente nas situações em que existe uma resposta inflamatória exacerbada ${ }^{21}$.

O alto consumo de ácido linoléico favorece o aumento do conteúdo de ácido araquidônico (AA) nos fosfolipídios das membranas celulares, aumentando, consequentemente, a produção de prostaglandina (PG) E2 e leucotrieno (LT) B4, por meio das vias enzimáticas da ciclooxigenase (COX) e 5-lipoxigenase (5-LOX), respectivamente. A ingestão de óleo de peixe introduz EPA nos fosfolipídios das membranas, inibindo o metabolismo do AA por competição pelas mesmas vias enzimáticas (COX e 5-LOX), promovendo a 


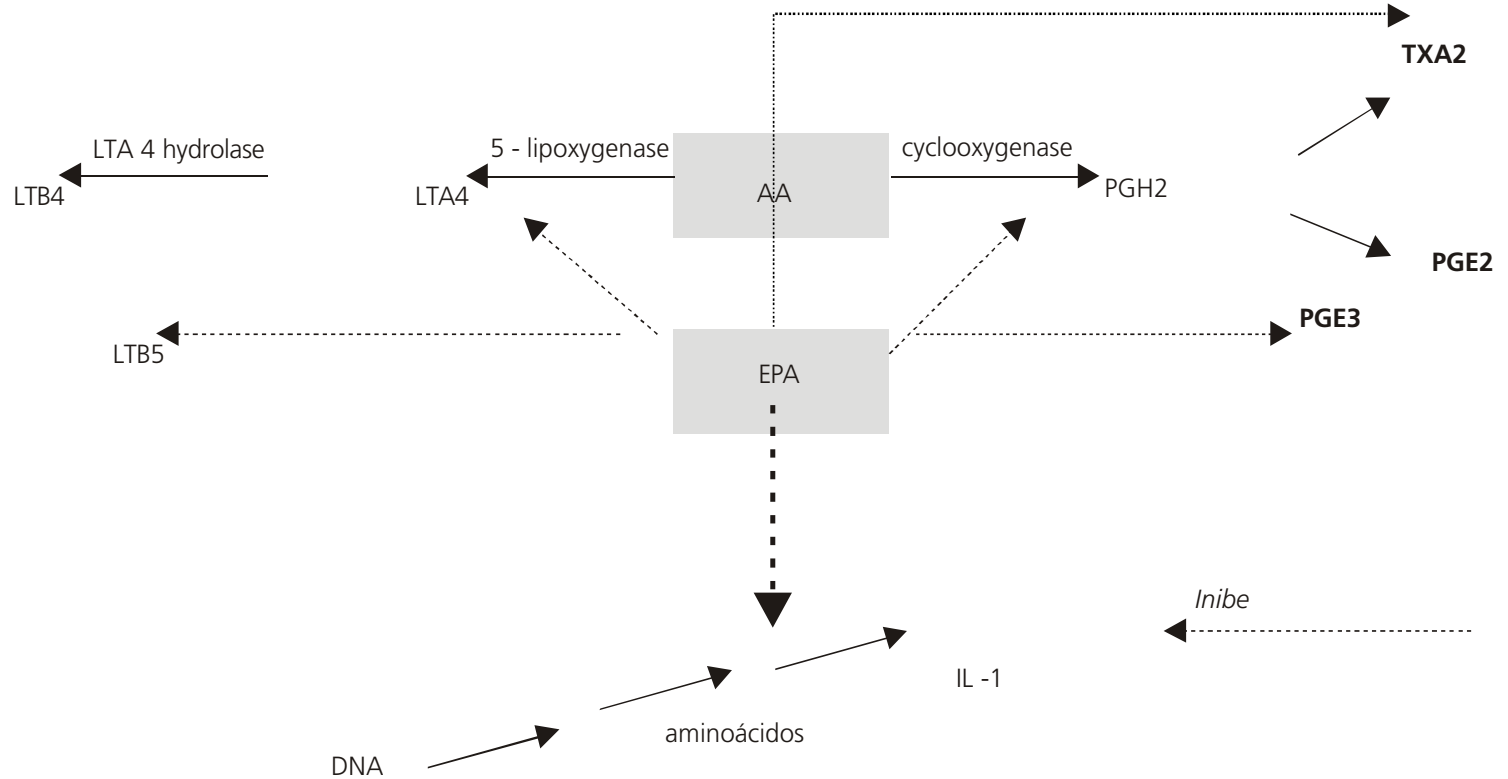

Figura 3. Metabolismo dos eicosanóides durante a produção de prostaglandinas, leucotrienos e tromboxanos por meio do AA e do EPA.

Adaptado de Calder $^{18}$ e James et al. ${ }^{22}$

formação de PGE3, em vez de PGE2, e LTB5, em vez de LTB4, que são mediadores inflamatórios menos ativos (Figura 3) ${ }^{24}$. Em geral, os cientistas concordam que o ácido linoléico é precursor da síntese de eicosanóides da série par, com características pró-inflamatórias, como o tromboxano $\mathrm{A} 2$ (TXA2), as PGI2 e PGE2 e os LTB44 ${ }^{24,25}$.

As PGE2 e os LTB4 são os mediadores que possuem o maior potencial pró-inflamatório. A PGE2 induz à febre, promove vasodilatação, aumenta a permeabilidade vascular e potencializa a dor e o edema causados por outros agentes, como bradicinina e histamina. Por outro lado, a PGE2 inibe a produção do TNF- $\alpha$ e IL-1, apresentando, nesse aspecto, característica antiinflamatória. Tem potencial imunossupressor, pois inibe a proliferação de linfócitos, a atividade das células natural killer (NK) e a produção de IL-2 e Interferon (IFN)- $\gamma$. O LTB4 aumenta a permeabilidade vascular, o fluxo sangüíneo e a quimiotaxia dos leucócitos, induz à liberação de enzimas lisossomais e aumenta a produção de espécies reativas de oxigênio e de TNF- $\alpha$, IL-1 e IL-6. Em todos esses aspectos, o LTB4 é pró-inflamatório ${ }^{19,26}$. Como citado, os tromboxanos (TX) também provêm do metabolismo dos eicosanóides. Entre eles, o TXA2 é o principal subproduto do $A A$, promovendo agregação plaquetária, adesão leucocitária e contração da musculatura lisa ${ }^{19}$.

Por outro lado, como apontado anteriormente, o aumento da oferta de ácidos graxos da família ômega-3, como o ácido linolênico (C18: 3) ou de EPA e de DHA, favorece a síntese de eicosanóides da série ímpar, como a PGE3, TXA3 e LTB5, que possuem características antiinflamatórias. Esse equilíbrio proporciona menor formação de mediadores pró-inflamatórios, reduzindo alguns dos efeitos imunosupressores (Figuras 2 e 3$)^{27}$.

Níveis de ingestão adequada (Al) de ácidos graxos essenciais foram estabelecidos pelo Institute of Medicine, por meio das Dietary Reference Intakes (DRIs), baseadas na ingestão média da população americana. Esses valores preconizados de consumo são de $17 \mathrm{~g}$ e $12 \mathrm{~g} / \mathrm{dia}$ de ácido linoléico ( $\omega$-6) e 1,6g e 1,1 g/dia de ácido linolênico ( $\omega-3)$ para homens e mulheres, respectivamente ${ }^{28}$. Por falta de dados suficientes, o Institute of Medicine não estabeleceu Al ou RDA para $A A, E P A$ ou $D H A^{22}$. 
Considerando que essas duas famílias de ácidos graxos competem pelas mesmas enzimas, o balanço entre $\omega$ - 6 e $\omega$-3 na dieta é de grande importância. Como conseqüência das mudanças no padrão dietético humano, a relação entre ácidos graxos $\omega-6: \omega-3$ na dieta também sofreu alterações no decorrer da História. No passado, na era paleolítica, essa relação contemplava, aproximadamente, $1: 1$ a 1:2, enquanto que o padrão atual (dieta ocidental) apresenta uma relação de 17:123. Embora alguns considerem satisfatória a relação $\omega-6: \omega-3$ de 10 a 5:128,29, a proposta mais recente, com base em experimentação animal, é de 1:123.

\section{Novas perspectivas na terapia nutricional com ômega-3}

Considerando os mecanismos descritos anteriormente, os efeitos da suplementação com ômega-3 vêm sendo investigados. Uma revisão recente apontou que a recomendação para o uso da suplementação com ômega-3 está bem estabelecida para determinadas condições clínicas, entre elas doença cardiovascular, hipertrigliceridemia e artrite reumatóide. Entretanto, outras condições, que podem se beneficiar da ação antiinflamatória do ômega-3, ainda estão sendo estudadas, apresentando evidências desses benefícios $27,30,31$.

Entre as ações que estão sendo estudadas, observam-se propriedades antineoplásica, imunossupressora e anticaquética ${ }^{32-34}$. Apenas as duas últimas serão abordadas na discussão a seguir.

Num estudo não randomizado, com o objetivo de verificar as repercussões da resposta inflamatória de 36 pacientes com câncer de pâncreas avançado, comparando-os com 6 indivíduos saudáveis, Barber et al. ${ }^{35}$ avaliaram as concentrações séricas de várias proteínas durante essa fase. Os autores observaram um aumento das proteínas positivas (proteína-C reativa, $\alpha$-1-ácido-glicoproteína, $\alpha$-1-antitripsina, haptoglobina e ceruloplasmina) e uma redução das proteínas negativas (albumina, transtirretina e transferrina) nos pacientes, em relação aos controles. Quando a suplementação com óleo de peixe $(2,18 \mathrm{~g}$ de EPA e $0,92 \mathrm{~g}$ de DHA) foi efetuada, observou-se aumento das proteínas negativas e redução das proteínas positivas em relação aos pacientes que receberam o suplemento padrão. Alterações significativas no peso corporal entre os dois grupos, com ganho no grupo que recebeu a suplementação com óleo de peixe, também foram observadas. Porém, como o peso pode sofrer variações em decorrência de fatores não relacionados ao estado nutricional em pacientes com câncer, esses resultados devem ser analisados com cautela.

A resposta inflamatória exacerbada em pacientes com câncer também pode promover uma utilização metabólica inadequada de substratos energéticos ${ }^{8}$. A fim de avaliar a oxidação desses substratos, em pacientes com câncer pancreático, e o impacto da suplementação com ômega-3, Barber et al. ${ }^{36}$ desenvolveram um ensaio clínico com suplementação de óleo de peixe $(2,2 \mathrm{~g}$ de EPA e $0,96 \mathrm{~g}$ de DHA) em 16 pacientes com câncer, comparando-os com 6 indivíduos saudáveis durante o jejum e no período alimentado. Para os pacientes com câncer foi oferecida uma suplementação com 2,2g de EPA e 0,96g de DHA por três semanas. Os autores observaram menor eficiência na oxidação de carboidratos nos pacientes, quando comparados aos controles saudáveis, evidenciada pela maior utilização de lipídios como fonte de energia, tanto no jejum, como no período alimentado. Porém, com a suplementação do óleo de peixe para os pacientes, observou-se uma resposta oxidativa mais adequada no metabolismo dos carboidratos, similar à dos controles.

Como citado anteriormente, pacientes com câncer também podem apresentar alterações no metabolismo dos lipídios, com aumento dos triglicérides circulantes pela redução da atividade da lipoproteína lipase (LPL), como conseqüência da resposta inflamatória ${ }^{4,6}$. A suplementação com ômega-3, em indivíduos saudáveis randomizados para receberem por quatro semanas, demons-trou reduzir os níveis pós-prandiais de triglicérides, bem como as apoproteínas B-48 e B-100 e a atividade da $\mathrm{LPL}^{37}$. 
Em um ensaio clínico randomizado duplo cego, a suplementação com óleo de peixe foi estudada, com o intuito de observar seu efeito na síntese de PGE2 de células mononucleadas do sangue periférico de pacientes pós-cirúrgicos com câncer, após estímulo endotoxêmico por Escherichia Coli. Dezoito pacientes receberam EPA $(2,74 \mathrm{~g})$ e DHA $(1,24 \mathrm{~g})$ por 7 dias. Após a intervenção, o grupo que recebeu suplementação com óleo de peixe apresentou menor produção de PGE2, em relação ao controle. Essa resposta com ômega-3 foi observada somente na vigência do estímulo endotoxêmico, sem alterações durante o período basal. Tais resultados confirmam o efeito benéfico do aumento do consumo de óleo de peixe, particularmente durante a resposta inflamatória intensa ${ }^{38}$.

Em outro ensaio clínico randomizado, Gogos et al. ${ }^{12}$ testaram a suplementação com ômega-3 (6 cápsulas por 3 vezes ao dia de $170 \mathrm{mg}$ de EPA e $115 \mathrm{mg}$ de DHA), em combinação com vitamina E por 40 dias versus placebo, na resposta imune e na sobrevida de pacientes gravemente doentes com vários tipos de câncer. Os autores demonstraram aumento significativo na relação Thelper/Tsupressor (CD4/CD8), tanto nos pacientes eutróficos, como nos desnutridos que receberam a suplementação. A porcentagem de sobrevida também demonstrou ser maior nos grupos de eutróficos e desnutridos randomizados para receberem a suplementação, do que nos grupos que não a receberam.

Em pacientes sépticos que necessitaram de nutrição parenteral, a suplementação randomizada com ômega-3 foi comparada ao uso da nutrição padrão com ômega-6. Os resultados demonstraram maior incorporação de EPA e DHA nos fosfolípides das membranas de leucócitos mononucleados nos pacientes que receberam o ômega-3, bem como uma menor síntese (ex-vivo) de citocinas pró-inflamatórias (TNF- $\alpha$, IL $1 \beta$ e IL-6) ${ }^{39}$.

Recentemente, um estudo retrospectivo analisou dados longitudinais de dois anos em pacientes que necessitaram de cirurgia abdominal de grande porte por várias causas, incluindo câncer. A amostra foi composta por três grupos: grupo 1 recebeu nutrição parenteral, sem óleo de peixe, no período pós-operatório (primeiro ano de estudo), grupo 2 recebeu nutrição parenteral pósoperatória com óleo de peixe e grupo 3 recebeu suplementação pré-operatória com óleo de peixe e nutrição parenteral pós-operatória com óleo de peixe, obtendo-se uma relação de 1:2 a 1:3 de ômega-3: ômega-6 (conduta com ômega-3 aplicada nos grupos 2 e 3, somente no segundo ano do estudo). Os resultados demonstraram menor tempo de hospitalização e redução nas taxas de mortalidade no grupo 3 e menor número de readmissões nos grupos 2 e 3, em relação aos controles, que não receberam óleo de peixe $\mathrm{e}^{40,41}$.

Em revisão recente, de 14 ensaios clínicos controlados, que testaram a suplementação com ômega-3, isolada ou combinada com arginina e/ou ácido ribonucléico (RNA) versus placebo, demonstrou-se redução do risco relativo para complicações após cirurgia de câncer em todos os pacientes ${ }^{42}$.

Os efeitos dos ácidos graxos sobre o metabolismo dos eicosanóides parecem ser dose-dependentes, demonstrando que elevações de pequena magnitude na PGE2 e no LTB4 têm a capacidade de causar um efeito estimulador sobre a produção de citocinas, aumentando a atividade do sistema imune, mas elevações maiores poderiam apresentar efeitos opostos ${ }^{25}$, predispondo o organismo a infecções.

Recentemente, demonstrou-se que a produção dos mediadores inflamatórios é determinada e regulada geneticamente, sendo a síntese do TNF- $\alpha$ regulada por várias unidades do genoma ${ }^{26}$. Tal característica determina a existência de indivíduos respondedores e não respondedores à suplementação com $\omega$-3. Essas diferentes respostas frente à suplementação com $\omega$-3 decorrem de mutações no gene do TNF, que podem produzir alelos do tipo TNF-2 e TNF- $\beta 2$. Essas modificações estão associadas ao aumento na produção de TNF- $\alpha$ e, portanto, à exacerbação 
da resposta inflamatória que, por sua vez, influencia no resultado clínico e na morbimortalidade. Alguns estudos têm demonstrado que indivíduos que possuem níveis mais altos de TNF- $\alpha$ antes da suplementação apresentam redução significante dos seus níveis após o consumo de ômega-3. Ao contrário, aqueles com níveis baixos, reagem com aumento de TNF- $\alpha$. Portanto, as respostas observadas entre os indivíduos podem ser atribuídas a diferenças nas características genéticas ${ }^{43}$.

Além disso, outras características do genótipo, que influenciam a produção de TNF- $\alpha$ e a resposta ao $\omega-3$, relacionam-se ao MHC (major histocompatibility complex) classe II, em particular à região HLA (human leukocyte antigens)-DR. Indivíduos que expressam HLA-DR15 são menos sensíveis à suplementação do que aqueles com HLA-DR3 e DR4, que, por esse motivo, apresentam respostas mais favoráveis ${ }^{26}$. Os resultados do estudo de Hughes et al. ${ }^{44}$ demonstraram redução na intensidade da expressão de todas as moléculas do MHC classe II (HLA-DR, HLA-DP, HLA-DQ), bem como das moléculas de adesão ICAM-1 (molécula de adesão intercelular-1) e LFA-1 (antígeno associado à função de leucócito-1).

Alguns estudos mostraram que a suplementação com ácidos graxos da família ômega-3 atenuou a resposta inflamatória aguda, prevenindo o catabolismo, e melhorou a utilização dos substratos energéticos, bem como a resposta imune, prolongando a sobrevida de pacientes com câncer. Entretanto, esses efeitos do óleo de peixe parecem estar mais associados com o EPA (Figura 4) ${ }^{32,45}$.

Embora existam evidências favoráveis da suplementação com $\omega$-3, outros estudos ${ }^{46}$ publicaram resultados contrários, em que os ácidos graxos poliinsaturados dessa família inibiram a resposta imune mediada por linfócitos T. Tal efeito é indesejável, principalmente em indivíduos previamente imunossuprimidos. Apesar dos resultados desses estudos, uma análise mais crítica sugere que a imunossupressão ocorreu, em parte, devido ao aumento da peroxidação lipídica pela oferta reduzida de antioxidantes, em particular de vitamina $E$, que pode ser prevenido com a sua suplementação ${ }^{47}$.

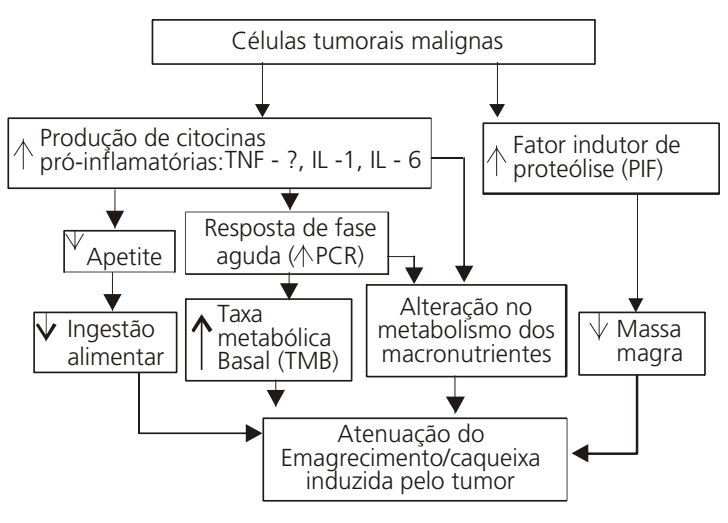

Figura 4. Mecanismo associado à caquexia e à perda de peso induzidas pelo tumor maligno.

PCR: proteína-C-reativa.

\section{CONSIDERAÇÕES FINAIS \\ E RE C O MEN D A ÇÕE S \\ PRÁTICAS PARA O USO DE $\omega-3$ E O $\omega-6$}

O aumento no consumo dietético de ácidos graxos poliinsaturados causa mudanças na composição dos ácidos graxos dos fosfolípides das membranas celulares. Essa alteração é responsável pelo aumento na fluidez da membrana, podendo interferir nas interações intracelulares, expressão de receptores de membrana, transporte de nutrientes e sinais de transdução, influenciando o crescimento celular ${ }^{25}$. Além disso, o aumento no número de ácidos graxos com várias duplas ligações pode tornar a membrana celular mais suscetível à peroxidação lipídica, se um aumento concomitante de nutrientes antioxidantes não for instituído. Por isso, recomenda-se um aumento na ingestão de $\alpha$-tocoferol para compensar 0 aumento no consumo de ácido graxo polinsaturado, a fim de estabilizar as duplas ligações. Entretanto, variações podem ocorrer, em função do grau de insaturação do lipídio, das diferentes quantidades depositadas nos tecidos e dos processos de elongação e dessaturaçãa ${ }^{48}$. É importante destacar que os ácidos graxos da família ômega-3 apresentam maior número de duplas ligações do que os das famílias ômega- 6 e 9 , o que os torna mais suscetíveis à peroxidação lipídica. Assim, alguns estudiosos acreditam que 
o consumo de produtos alimentares com alto teor de ácidos graxos poliinsaturados deve ser acompanhado de, no mínimo, 0,4mg a 0,6mg de vitamina $E$ por grama de ácido graxo polinsaturado $^{29,48}$.

As principais fontes de ácido $\alpha$-linolênico (18:3) são os óleos de canola $(9,3 \mathrm{~g} / 100 \mathrm{~g})$ e soja $(2,6 \mathrm{~g} / 100 \mathrm{~g})$ e a noz $(6,8 \mathrm{~g} / 100 \mathrm{~g})$, e as principais fontes de EPA e DHA são salmão $(0,84$ e 0,81 g/100g), sardinha $(0,47$ e 0,51g/100g), caviar $(1,03$ e $1,35 \mathrm{~g} / 100 \mathrm{~g})$ e ostra $(0,42$ e $0,46 \mathrm{~g} / 100 \mathrm{~g})$. A gema do ovo contém uma pequena quantidade desses ácidos $(0,01 \text { e 0,11 g/100g })^{49}$.

Questões associadas às inter-relações entre ácidos graxos ômega-3 e ômega-6 ainda necessitam ser estudadas. Permanece obscuro para qual extensão o ácido linolênico é convertido em EPA e DHA no organismo humano, e se tal conversão varia com a idade ou estado fisiológico. Além disso, também não se sabe qual a extensão do impacto da ingestão de ômega- 6 sobre a taxa de conversão do ômega-3 em EPA e DHA, ou se tal ingestão causa alterações nos efeitos biológicos atribuídos ao EPA e DHA ${ }^{22}$.

Efeitos colaterais relacionados à suplementação com ômega-3 não demonstraram alterações relevantes. Entre tais efeitos poderia ocorrer aumento nos níveis de LDL-colesterol, aumento no tempo de sangramento e piora do perfil glicêmico em diabéticos. Suplementações consideradas seguras são de até $16 \mathrm{~g}$ de óleo de peixe por dia. Porém, recomenda-se monitoramento em indivíduos que recebem doses superiores a $3 \mathrm{~g}$ por $\mathrm{dia}^{27}$.

Consideração adicional deve ser feita quanto à condição clínica do paciente, quando se decide efetuar uma suplementação com ômega-3. É importante destacar que durante estados mórbidos mais graves, como infecções, sepse e falência orgânica múltipla, por exemplo, o organismo reage por meio de uma resposta inflamatória bifásica, caracterizada pela produção de diferentes citocinas. A primeira fase, hiperinfla- matória - systemic inflammatory response syndrome (SIRS), é caracterizada por hiperatividade dos leucócitos e liberação de citocinas pró-inflamatórias, como IL-1, IL-6 e TNF- $\alpha$. Na segunda fase, hipoinflamatória compensatory anti response syndrome(CARS), há redução da capacidade dos leucócitos em destruir microrganismos, devido à exacerbação da resposta antiinflamatória, aumentando o risco de infecção secundária. Nessa fase, há produção de IL-4, IL-10, IL-13 e TGF- $\beta$, que são citocinas de caráter antiinflamatório ${ }^{50}$.

Portanto, o ômega-3 pode controlar a resposta hiperinflamatória exacerbada, minimizando os efeitos dos distúrbios metabólicos, o hipercatabolismo e, desse modo, melhorar o prognóstico. Entretanto, se oferecido durante a fase hipoinflamatória, possivelmente irá expor o paciente ao risco de infecção secundária, pois, como citado, reduz a resposta inflamatória, podendo atuar diminuindo a atividade imunológica de forma inadequada ${ }^{51}$. Assim, o uso desse imunomodulador deve ser criterioso, particularmente em pacientes com doença grave.

Os estudos envolvendo essas áreas de conhecimento são difíceis de realizar, considerando a complexidade do sistema imune e das respostas envolvidas durante a inflamação. Os resultados controversos se devem, provavelmente, a esses fatores, bem como à variabilidade interindividual e às diferenças na metodologia dos estudos.

Mesmo os resultados de algumas metanálises e reviões, como as realizadas para medir o efeito do ômega-3 em transplante de órgãos e no câncer ${ }^{27,42}$, podem apresentar vieses, se considerada a possibilidade de que estudos com resultados negativos ao uso da suplementação tendem a ser omitidos das publicações, devido, principalmente, ao seu impacto desfavorável para a indústria.

Finalmente, ainda existem poucos estudos em seres humanos comprovando os efeitos benéficos dessa terapia em pacientes com câncer durante o tratamento antineoplásico. Esses são necessários para determinar se a modulação 
desses lipídios poderia atenuar a resposta pró-inflamatória nesses indivíduos e, portanto, favorecer a resposta nutricional e a farmacocinética e farmacodinâmica das drogas, melhorando o prognóstico de cura.

Áreas para futuras pesquisas incluem ensaios de prevenção primária, nível ótimo de ingestão entre ômega-6, ômega-3, EPA e DHA e estudos sobre as várias indicações clínicas, bem como o aprimoramento das informações sobre a segurança e confiança nos produtos consumidos, quer seja alimento ou suplemento.

\section{REFERÊNCIAS}

1. Wong PW, Enriquez A, Barrera R. Nutritional support in critically ill patients with cancer. Crit Care Clin. 2001; 17(3)

2. Harrison LE, Brennan MF. The role of total parenteral nutrition in the patient with cancer. Curr Prob Surg. 1995; 32(10):833-924.

3. Garófolo A. Estado nutricional de crianças e adolescentes com câncer [dissertação]. São Paulo: Escola Paulista de Medicina, Universidade Federal de São Paulo; 2000

4. Holcomb GW, Ziegler MM. Nutrition and cancer in children. Surg Ann. 1990; 129-41.

5. Mauer AM, et al. Special nutrition needs of children with malignancies: a review. JPEN. 1990; 14:315-24.

6. Laviano A, Meguid MM, Yang ZJ, Gleason JR, Cangiano C, Fanelli FR. Cracking the riddle of cancer anorexia. Nutrition. 1996; 12:706-10.

7. Keefe DMK, Cummins AG, Dale BM, Kotasek D, Robb TA, Sage E. Effect of high-dose chemotherapy on intestinal permeability in humans. Clin Sci. 1997; 92:385-9.

8. Tisdale MJ. Cancer caquexia: metabolic alterations and clinical manifestations. Nutrition. 1997; 13:1-7.

9. Kyle UG, Pirlich M, Schuetz T, Lochs H, Pichard C. Is nutritional depletion by nutritional risk index associated with increased length of hospital stay? A population-based study JPEN. 2004; 28(2): 99-104.

10. Windsor JA, Graham LH. Weight loss with physiologic impairment. A basic indicator of surgical risk. Ann Surg. 1987; 207(3):290-6.

11. Villa ML, Ferrario E, Bergamasco E, Bozzetti F, Cozzaglio L, Clerici E. Reduced natural killer cell activity and IL-2 production in malnourished cancer patients. Br J Cancer. 1991; 63:1010-4.
12. Gogos CA, Ginopoulos P, Salsa B, Apostolidou E, Zoumbos NC, Kalfarentzos F. Dietary omega-3 polyunsaturated fatty acids plus vitamin E restore immunodeficiency and prolong survival for severely ill patients with generalized malignancy. A randomized control trial. Cancer. 1998; 82(2): 395-401.

13. Garófolo A, Lopez FA. Novos conceitos e propostas na assistência nutricional da criança com câncer. Rev Paul Pediatr. 2002; 20:140-6.

14. Torosian $\mathrm{MH}$. Cancer cachexia and nutrition support in the cancer patient. A clinical review. Cancerología. 1993; 39:1768-74.

15. Tchekmedyian SN, Halpert C, Ashley J, Herber D. Nutrition in advanced cancer: anorexia as a outcome variable and target of therapy. JPEN. 1992; 16(6):88S-92S.

16. Tisdale MJ. Cancer anorexia and cachexia. Nutrition. 2001; 17:438-42.

17. Cohen J, Lefor AT. Nutrition support and cancer. Nutrition. 2001; 17:698-9.

18. Slaviero KA, Clarke SJ, Rivory LP. Inflammatory response: an unrecognized source of variability in the pharmacokinetics and pharmacodynamics of cancer chemotherapy. Lancet Oncol. 2003; 4(4):224-32

19. Calder PC. Long-chain n-3 fatty acids and inflammation: potential application in surgical and trauma patients. Braz J Med Biol Res. 2003; 36(4):433.

20. Bistrian BR. Clinical aspects of essential fatty acid metabolism: Jonathan Rhoads lecture. JPEN. 2003: 27(3):168.

21. Thompson WA, Lowry SF. Effect of nutrition on inflammatory mediators. In: Zaloga GP. Nutrition in critical care. St Louis: Mosby; 1994. p.505-23.

22. Agency for Healthcare Research and Quality. Effects of omega-3 fatty acids on organ transplantation. Department of Health and Human Services; 2005. p.115.

23. Fürst $P$. The striking diet of the island of Crete: lipid nutrition from the palaeolithic to the affluent modern society. Clin Nutr. 2002; 21(S2):9-14.

24.James MJ, Gibson RA, Cleland LG. Dietary polyunsaturated fatty acids and inflammatory mediator production. Am J Clin Nutr. 2000; 71(S):343-8.

25. Kelley DS. Modulation of human immune and inflammatory responses by dietary fatty acids. Nutrition. 2001; 17:669-73.

26. Grimble R. Use of n-3 fatty acid-containing lipid emulsions in the Intensive Care Unit environment: the scientist's view. Clin Nutr. 2002; 21(S2):15-21. 
27. Robert O. Practical applications of fish oil (w-3 fatty acids) in primary care. J Am Board Pract. 2005; 18:28-36.

28. Institute of Medicine. Dietary Reference Intakes (DRIs) for energy, carbohydrate, fiber, fat, fatty acids, cholesterol, protein, and amino acids. Part 1. Washington (DC): National Academy Press; 2002.

29. Nutrition and Health Collection. Danone Research Center. Nutritional recommendations. Lipids from alpha to omega. Paris: John Libbey Eurotext; 1999.

30. Lasztity N, Hamvas J, Biro L, et al. Effect of enterally administrad $n-3$ polynsaturated fatty acids in acute pancreatitis-a prospective radomized clinical trial. Clin Nutr. 2005; 24:198-205.

31. Meier R. Enteral fish oil in acute pancreatitis. Clin Nutr. 2005: 24:169-71.

32. Barber MD. Cancer caquexia and its treatment with fish-oil-enriched nutritional supplementation. Nutrition. 2001; 17:751-5.

33. Fearon $\mathrm{KCH}$. The anticancer and anticachetic effects of n-3 fatty acids. Clin Nutr. 2002; 21(S2):73-7.

34. Hardmam WE. Omega-3 fatty acid to augment cancer therapy. J Nutr. 2002; 132:3508S-12S.

35. Barber MD, Ross JA, Preston T, Shenkin A, Fearon $\mathrm{KCH}$. Fish oil-enriched nutritional supplement attenuates progression of the acute-phase response in weight-losing patients with advanced pancreatic cancer. J Nutr. 1999; 129:1120-5.

36. Barber MD, McMillan DC, Preston T, Ross JA, Fearon $\mathrm{CH}$. Metabolic response to feeding in weight-losing pancreatic cancer patients and its modulation by a fish-oil-enriched nutritional supplement. Clin Sci. 2000; 98:389-99.

37. Park Y, Harris WS. Omega-3 fatty acid supplementation accelerates chylomicron triglyceride clearence. J Lipid Res. 2003; 44(3): 455-63.

38. Swails WS, Kenler AS, Driscoll DF, et al. Effect of a fish oil structured lipid-based diet on prostaglandin release from mononuclear cells in cancer patients after surgery. JPEN. 1997; 21(5): 266-74.

39. Mayer K, Gokorsch S, Fegbeutel C, et al. Parenteral nutrition with fish oil modulates cytokine response in patients with sepsis. Am J Respir Crit Care Med. 2003; 167(10):1321-8.

40. Tsekos E, Reuter C, Stehle P, Boeden G. Perioperative administration of parenteral fish oil supplements in a routine clinical setting improves patient outcome after major adbominal surgery. Clin Nutr. 2004; 23(3):325-30.
41. Tsekos E, Reuter C, Stehle P, Boeden G. Corrigendum to "Perioperative administration of parenteral fish oil supplements in a routine clinical setting improves patient outcome after major adbominal surgery". Clin Nutr. 2004; 23(4): 755-6.

42. Maclean CH, Newberry SJ, Mojica WA, et al. Effects of Omega-3 fatty acids on cancer. Evid Rep Technol Assess. 2005; 113(summ):1-4.

43. Grimble RF, Howell WM, O'Reilly, et al. The ability of fish oil to suppress tumor necrosis factor a production by peripheral blood mononuclear cells in healthy men is associated with polymorphisms in genes that influence tumor necrosis factor a production. Am J Clin Nutr. 2002; 76(2):454-9.

44. Hughes DA, Pinder AC, Piper Z, Johnson IT, Lund EK. Fish oil supplementation inhibits the expression of major histocompatibility complex class II molecules and adhesion molecules on human monocytes. Am J Clin Nutr. 1996; 63(2):267-72.

45. Beck AS, Smith KL, Tisdale MJ. Anticachetic and antitumor effect of eicosapentaenoic acid and its effect on protein turnover. Cancer Res. 1991; 51(15):6089-93.

46. Thies F, Nebe-von-Caron G, Powell JR, Yaqoob P, Newsholme EA, Calder PC. Dietary supplementation with eicosapentaenoic acid, but not with other long-chain n-3 or n-6 polyunsaturated fatty acids, decreases natural killer cell activity in healthy subjects aged $>55 \mathrm{y}$. Am J Clin Nutr. 2001; 73(3):539-48.

47. Wu D, Meydani SN. N-3 polyunsaturated fatty acids and immune function. Proc Nutr Soc. 1998; 57(4):503-9.

48. Institute of Medicine. Dietary reference intakes (DRIs) for vitamin C, vitamin E, selenium, and carotenoids. Washington (DC): National Academy Press; 2000.

49. Bloch AS, Shils ME. Appendix. In: Shils ME, Olson JA, Shike M. Modern nutrition in health and disease. 8th ed. Malvern: Lea \& Febiger; 1994. p.A100-4.

50. Grimminger F, Seeger W, Mayer K. Use of n-3 fatty acid-containing lipid emulsions in the intensive care unit environment: the clinician's view. Clin Nutr. 2002; 21(S2):23-9.

51. Pablo MA, Puertollano MA, Cienfuegos GA. Biological and clinical significance of lipids as modulators of immune system functions. Clin Diagn Lab Immunol. 2002; 9(5):945-50.

Recebido em: 26/7/2004

Versão final reapresentada em: 16/6/2005 Aprovado em: 20/9/2005 